\title{
Hematopoietic stem and progenitor cells in HIVIAIDS and immune reconstitution
}

\author{
Jielin Zhang ${ }^{1}$, Clyde S Crumpacker ${ }^{1,2}$ \\ ${ }^{I}$ Department of Medicine at Brigham and Woman's Hospital, Boston, MA 02115, USA; ${ }^{2}$ The Division of Infectious Diseases, Beth \\ Israel Deaconess Medical Center, Harvard Medical School, Boston, MA 02215, USA \\ Cell Research (2010) 20:745-747. doi:10.1038/cr.2010.85; published online 22 June 2010
}

The human immunodeficiency virus type 1 (HIV-1) causes an acquired immunodeficiency syndrome (AIDS). HIV-1 infects human immune cells, specifically $\mathrm{CD}^{+}$lymphocytes, which leads to AIDS and undermines reconstitution of immunity. The unique challenges of HIV/AIDS have triggered multidisciplinary investigators to study the virology of the pathogen and the biology of the host cells, especially the interactions of HIV-1 with T-lymphocytes, macrophages, and hematopoietic stem and progenitor cells (HSPC) [1-8].

To study the possibility that HIV-1 can infect multipotent progenitor cells and cause cell death and latent infection in cellular reservoirs, Carter et al. [9] have used different clones of the DNA plasmid, p89.6 to infect $\mathrm{CD} 34^{+}$multipotent hematopoietic progenitor cells (HPCs). They have found that some HPCs were infected in cell culture and rapidly killed. Carter et al. conclude that these findings enhance the understanding of HIV bone marrow pathology and the mechanisms by which HIV causes persistent infection.

\section{Are HPCs susceptible to HIV in- fection?}

Correspondence: Jielin Zhanga, Clyde S Crumpacker $^{\mathrm{b}}$

aE-mail: jlzhang@partners.org

bE-mail: ccrumpac@bidmc.harvard.edu
It is generally accepted that human hematopoietic stem cells (HSCs) are $\mathrm{CD} 34^{+} \mathrm{CD} 38^{\mathrm{lo} /}-$ primary bone marrow cells. As HSCs represent only $0.5-3 \%$ of mononuclear cells in human bone marrow, researchers have used $\mathrm{CD} 34^{+}$ primary bone marrow cells without a further selection of the CD38 marker nor test of cell function with long-term culture-initiating cell assay (LTC-IC) $[1-4,10-13]$. These CD $34^{+}$primary hematopoietic stem and progenitor cells are dubbed HSPC [1-4, 10-13]. The multipotent progenitor cells, however, are named MPPs, and these cells possess multipotentiality but have a function for limited self-renewal (Figure 1). Beside $\mathrm{CD} 34^{+}$, MPPs are $\mathrm{CD} 150^{-}$ CD48-CD244+.

Carter et al. have used cells designating HPCs in their study, meaning CD34 ${ }^{+}$multipotent HPCs, similar to the MPPs. These HPCs, however, appear to be HSPC, as no assay results

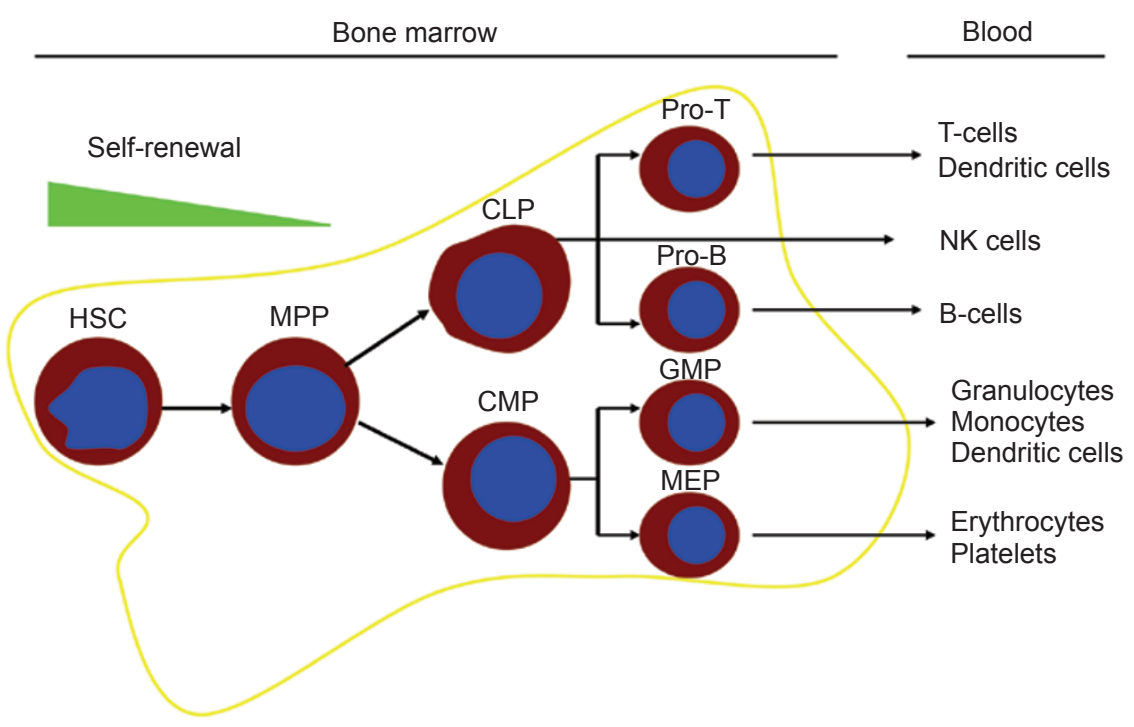

Figure 1 Hierarchy of human hematopoiesis. HSC, hematopoietic stem cells; MPP, multipotent progenitor; CLP, common lymphoid progenitor; CMP, common myeloid progenitor; GMP, granulocyte/macrophage progenitor; MEP, megakaryocyte/erythrocyte progenitor. 
are shown that these cells are either HSCs that are $\mathrm{CD} 34^{+} \mathrm{CD} 38^{\text {lo/ }-}$ or MPPs that are $\mathrm{CD} 34^{+} \mathrm{CD} 150^{-} \mathrm{CD} 48^{-} \mathrm{CD} 244^{+}$. HIV-1 infection of HSPC has been studied since the 1980s. If the HSPC are purely isolated and maintained under an unactivated undifferentiated culture condition, HSPC are resistant to HIV-1 infection [1-3, 5-8]. Carter et al. have indicated that they have used $\mathrm{CD} 133^{+}$ cells. If the cells used in their molecular assays are a subgroup of HSPC that are $\mathrm{CD} 133^{+} \mathrm{CD} 34^{+} \mathrm{CD} 38^{\text {lo/- }}$, the studies of Carter et al. on these cells may provide a different result from that previously reported on the HIV-1 infection of HSPC. Carter et al., however, have not precisely defined whether they have used a subset of $\mathrm{CD} 34^{+}$bone marrow cells in their in vitro assays, nor have they consistently used these cells in the HIV-1 infection studies, neither in the sections of results nor methods.

\section{HIV actively infects HPCs}

In their study, Carter et al. have used 7 vector constructs of 89.6 to make the pseudo-viruses of 89.6. They have not specified, however, whether they have used the HIV-1 89.6 virus in the studies of HIV infection, even for one of the HIV-1 infection assays. The HIV-1 89.6 virus is different from the p89.6 clone, the former being an HIV-1 RNA virus, and the latter a DNA clone of HIV-1 89.6. Despite using 7 clones, the vectors or the pseudo-viruses used in their infection assays apparently all contain a full-length gag that encodes the most abundant protein found in an HIV-1 virion, the HIV-1 Gag. HIV-1 Gag is a packaging protein which encapsulates the viral genome and is not removed (or uncoated) from the viral genome until the steps after entry.

The criterion for HIV-1 infection of HPCs employed by Carter et al. was to detect the intra cellular Gag after a spin infection. The pseudo-viruses have no difficulty in entering HPCs following the spin infection and they accomplish this more easily than a routine infection with the HIV-1 virus [1-7]. The HIV-1 Gag in the pseudo-viruses can be easily detected with flow cytometry without a further expression of gag gene from the pseudo-viruses in the cell. Such pseudo-viruses have been extensively used by others to carry out a single cycle infection, since after entering the cells, these pseudo-viruses cannot further infect or spread to other cells. Furthermore, the detected Gag could be from exracellular pseudo-virus that is sticking to the cells.

To confirm that the HIV-1 virus actively infects HPCs, another method should also be employed to measure the active replication of HIV-1 in cells, rather than relying on detection of intra cellular Gag as the sole criterion to conclude that HIV actively infects HPCs. Other methods and techniques for detecting HIV-1 active infection of cells could include the determination of a late gene expression in the viral lifecycle such as HIV-1 env, or a logarithmic increase of Gag p24 protein in the culture supernatant but not in cells. No other confirmatory measures of active HIV-1 infection, however, were employed by Carter et al. to show that HIV-1 actively infects HPCs from Figures 1 to 3 titled "HIV infects ... HPCs" and "Multipotent HPCs are susceptible to HIV infection".

\section{Evidence that $\mathrm{CD34}^{+}$bone mar- row cells are infected in vivo}

An important goal of bench experiments is to reveal the molecular mechanisms of whether HIV-1 is infective or not for HPCs (or i.e. HSPC) and to provide insight into controlling HIV-1 infection and reconstituting patient immunity. Carter et al. have examined the HPCs samples of 15 patients in their study, by the method previously employed to detect intracellular Gag after the spin infection plus a detection of HIV DNA.

The positive rate of $\mathrm{CD} 34^{+} \mathrm{Gag}^{+}$cells from these 15 patients apparently depends on the purity of the cell samples. Carter et al. have shown that if the sample purity is $98-85 \%$, the positive rate of $\mathrm{CD} 34^{+} \mathrm{Gag}^{+}$cells is $0 \%$. If the sample purity is $62 \%$, the positive rate of cells is $0.9 \%$. If the sample purity is $41 \%$, the positive rate of cells is $1.8 \%$. And finally, if the sample purity is down to $19 \%$, the positive rate of cells is increased to $4.8 \%$. The correlation of a higher positive rate of $\mathrm{CD} 34^{+} \mathrm{Gag}^{+}$ cells with decreasing cell purity raises the distinct possibility that the apparent positive rate is due to contamination with other cell types.

As for detection of HIV DNA, Carter et al. report that HIV DNA was detected in $\mathrm{CD} 34^{+}$cells from 4 of 9 patients who were on HAART and had an undetectable viral load for longer than 6 months. Shown in the supplementary Table 1, however, these patients were negative for intracellular $\mathrm{Gag}$ in $\mathrm{CD} 34^{+}$cells despite presence of HIV DNA. Furthermore, for the 6 patients who had a positive viral load in the Table 1, only 3 of them were positive for intra cellular Gag. Since the intracellular $\mathrm{Gag}^{+}$were detected from the samples of only 3 of the total 15 patients, the positive detection of Gag is $3 / 15$ but not $3 / 6$ as the authors stated. Additionally, the authors do not indicate why these 6 patients were not tested for HIV DNA in their $\mathrm{CD} 34^{+}$cells. The data on the detection of HIV DNA on all 15 patients should have been included in the supplementary Table 1 , and it is misleading not to do so. A much lower positive rate of $\mathrm{Gag}^{+} \mathrm{CD} 34^{+}$cells is found in patient samples, which is not in agreement with the in vitro results where pseudo-viruses were used to infect HPCs in culture and Gag was detected in HPCs as the only criterion employed to show that HIV actively infects HPCs.

To provide a balanced view of the results presented by Carter et al., additional studies on patient samples will be needed. It will be important to correlate the presence of integrated HIV DNA 
in cells with detection of intracellular $\mathrm{Gag}^{+}$expression. It is unexplained in the study of Carter et al. why $\mathrm{Gag}^{+} \mathrm{CD} 34^{+}$ cells were detected in only 3 of 6 freshly isolated samples from patients with high viral loads and why the integrated HIV DNA data on these patients is not provided.

Other inconsistent results are present in the study of Carter et al., such as in the HIV pathology of bone marrow. It has been known that besides the $\mathrm{CD} 34^{+}$cells, bone marrow harbors the mononuclear progeny cells that are newly differentiated from HSPC. These include $\mathrm{T}$ - and $\mathrm{B}$-lineage cells and bone marrow stromal cells [5-8, 10-13]. The infection of these cells has been previously reported to play important roles in HIV-1 pathology in the bone marrow [8]. Carter et al. report that bone marrow mononuclear cells immunodepleted for $\mathrm{CD}_{34}{ }^{+}$cells did not release HIV and rapidly died. This may require additional studies to define whether these cells were negative for the intracellular Gag or were negative for release of HIV. The exact reason why the bone marrow mononuclear cells, which were immunodepleted for $\mathrm{CD} 34^{+}$cells, did not release HIV and died rapidly needs to be identified. These cells appear to behave similarly to the HPCs that died rapidly but were actively infected by p89.6 in the same experiments.

\section{A role of HSPC in HIV/AIDS and renewal of immune function}

More studies remain to be performed to understand the molecular mechanism by which HIV-1 causes immunodeficiency. The role that HSPC play in this mechanism is of a particular interest. Much significant progress has been achieved in the past, including the isolation of HIV-1, virus cultivation in cell culture in vitro, identification of the HIV-1 lifecycle, and development of highly active anti-retroviral therapy (HAART). A final achievement will be finding the residual viral reservoirs and possibly eradicating HIV-1 infection and renewing a patient's immune function.

As HSPC are the best-studied human stem and progenitor cells, researchers have put a lot of effort to harness the power of HSPC in treatment of HIV/ AIDS and in the reconstitution of patient immunity. Methods to detect the shortlived human HSCs are not yet available and it remains an important goal to be able to identify these cells. Defining the relationship of HIV-1 infection of HSPC and the role of HSPC in immune reconstitution is undoubtedly important. Researchers must know the limitations and strengths of the techniques and methods in virology and stem cell biology that they employ. Finding ways to utilize and harness the HSPC to treat HIV/AIDS and to reconstitute immunity has become imperative. This requires careful study design from bench to the bedside, and correct, objective interpretation of results.

\section{References}

1 Shen H, Cheng T, Preffer FI, et al. Intrinsic human immunodeficiency virus type 1 resistance of hematopoietic stem cells despite coreceptor expression. $J$ Virol 1999; 73:728-737.

2 Zhang J, Scadden DT, Crumpacker CS. Primitive hematopoietic cells resist HIV-1 infection via p21. J Clin Invest 2007; 117:473-481.

3 Zhang J, Attar E, Cohen K, Crumpacker C, Scadden D. Silencing p21(Wafl/ Cip1/Sdi1) expression increases gene transduction efficiency in primitive human hematopoietic cells. Gene Ther 2005; 12:1444-1452.

4 Luo XM, Maarschalk E, O’Connell
RM, Wang P, Yang L, Baltimore D. Engineering human hematopoietic stem/ progenitor cells to produce a broadly neutralizing anti-HIV antibody after in vitro maturation to human B lymphocytes. Blood 2009; 113:1422-1431.

5 Weichold FF, Zella D, Barabitskaja O, et al. Neither human immunodeficiency virus-1 (HIV-1) nor HIV-2 infects most-primitive human hematopoietic stem cells as assessed in long-term bone marrow cultures. Blood 1998; 91:907-915.

6 von Laer D, Hufert FT, Fenner TE, et al. $\mathrm{CD} 34+$ hematopoietic progenitor cells are not a major reservoir of the human immunodeficiency virus. Blood 1990; 76:1281-1286.

7 Folks TM, Kessler SW, Orenstein JM, Justement JS, Jaffe ES, Fauci AS. Infection and replication of HIV-1 in purified progenitor cells of normal human bone marrow. Science 1988; 242:919922

8 Stutte HJ, Müller H, Falk S, Schmidts HL. Pathophysiological mechanisms of HIV-induced defects in haematopoiesis: pathology of the bone marrow. Res Virol 1990; 141:195-200.

9 CarterCC, Onafuwa-NugaA, McNamara LA, et al. HIV-1 infects multipotent progenitor cells causing cell death and establishing latent cellular reservoirs. Nat Med 2010; 16:446-451.

10 Jaiswal S, Weissman IL. Hematopoietic stem and progenitor cells and the inflammatory response. Ann N Y Acad Sci 2009; 1174:118-121.

11 Ueno H, Weissman IL. Stem cells: blood lines from embryo to adult. $\mathrm{Na}$ ture 2007; 446:996-997.

12 Bhattacharya D, Czechowicz A, Ooi AG, Rossi DJ, Bryder D, Weissman IL. Niche recycling through divisionindependent egress of hematopoietic stem cells. J Exp Med 2009; 206:28372850.

13 Kondo M, Wagers AJ, Manz MG, et al. Biology of hematopoietic stem cells and progenitors: implications for clinical application. Annu Rev Immunol 2003; 21:759-806. 